\title{
Nanoscale Phase Patterning in a Sr-Doped Lanthanum Cobaltite Thin Film
}

\author{
D.N. Leonard ${ }^{1}$, K. Klein ${ }^{2}$, D.A. Cullen ${ }^{1}$ \\ ${ }^{1}$ Materials Science \& Technology Div., Oak Ridge National Laboratory, Oak Ridge, TN 37831 USA \\ ${ }^{2}$ Carl Zeiss NTS, Peabody, MA 01960 USA
}

Increasing the energy density and efficiency of intermediate temperature (e.g. $500^{\circ} \mathrm{C}-800^{\circ} \mathrm{C}$ ) solid oxide fuel cells (SOFCs) remains one of the barriers to increased applicability of these devices as an alternative energy source. Recently, increased electrocatalytic activity at moderate temperature $\left(\sim 520^{\circ} \mathrm{C}\right)$ was reported for thin film multilayers of perovskite lanthanum strontium cobaltite $\left(\mathrm{La}_{0.8} \mathrm{Sr}_{0.2} \mathrm{CoO}_{3}-\mathrm{LSC}_{113}\right)$ and Ruddlesden-Popper-like $\mathrm{LaSrCoO}_{4}-\mathrm{LSC}_{214}$ [1]. During SOFC operation, changes in cathode microstructure, e.g., crystallization, amorphization, [2] and cation segregation, [3] can occur, impacting cathode activity [4]. Here, we demonstrate nanoscale patterning of amorphous regions in a crystalline thin film $\mathrm{LSC}_{113}$ cathode.

Epitaxial $65 \mathrm{~nm}$ thick films of $\mathrm{LSC}_{113}$ were grown on the yttria-stabilized zirconia (YSZ) substrate with intermediate Gd-doped $\mathrm{CeO}_{2}$ (GDC) buffer layer by pulsed laser deposition. Patterning of amorphous regions in the crystalline matrix was accomplished with a focused He ion beam; three rectangular areas were patterned, measuring $0.2 \mu \mathrm{m} \times 1 \mu \mathrm{m}$, with varying He ion doses (Table 1). Following the lithographic implantation, a dual beam SEM/FIB was used to extract the implanted regions. These extracted sections were FIB-thinned to electron transparency. The cross-sections were examined using aberration corrected scanning transmission electron microscopy (STEM) in high angle annular dark field (HAADF) mode, electron energy loss spectroscopy (EELS), and energy dispersive spectroscopy (EDS).

High-dose implanted regions (A and C, Table 1) showed a topographic change in the surface of the $\mathrm{LSC}_{113}$ thin film, as shown in the secondary electron (SE) image (Fig. 1). Figures 2(a-c) show crosssectional HAADF-STEM images of the three implanted regions. A swelling of the $\mathrm{LSC}_{113}$ thin film (of about 10nm) is evident for the material implanted at the higher dose of $0.12 \mathrm{nC} / \mu \mathrm{m}$ (regions $\mathrm{A}$ and $\mathrm{C}$, Fig. 1). The swelling was associated with amorphization of the $\mathrm{LSC}_{113}$. This amorphization is highly localized: fft diffractograms (inset, Fig. 3) show that the implanted region A is amorphous, while a region directly adjacent to it is fully crystalline, suggesting the feasibility of nanopatterning to alter the thin film cathode activity. The width of the patterned amorphous region was slightly larger for a $30 \mathrm{kV}$ beam (region A, Table 1). EDS analysis shows that the $\mathrm{La}, \mathrm{Sr}, \mathrm{Co}$, and O content of the amorphous and crystalline regions is the same, thus He-beam-induced amorphization is not accompanied by chemical segregation. Higher magnification HAADF-STEM imaging (see Fig.4) showed that bubbles also formed throughout the implanted region of the $\mathrm{LSC}_{113}$; these bubbles contain He, as determined by EELS. Interestingly, the GDC buffer and YSZ substrate remained crystalline with no evidence for He bubble formation; in fact the $\mathrm{LSC}_{113} / \mathrm{GDC}$ interface showed a marked increase in the number of He bubbles compared to adjacent regions, suggesting that $\mathrm{He}$ diffusion across that interface is hindered (Fig. 4). This "bubble pile-up" is best observed by the intensity profile of the HAADF-STEM image (inset, Fig. 4). The observed difference in the He beam dose-tolerance and penetrability between $\mathrm{LSC}_{113}$ and GDC/YSZ suggests the possibility of chemically selective nanopatterning; the origins of this behavior will be discussed [5]. 
References:

[1] E.J. Crumlin, et al., J. Phys. Chem. Lett. 1, 3149-3155 (2010).

[2] M. Kubicek, et al., J. Electrochem. Soc. 158, B727-B734 (2011).

[3] J. Januschewsky, et al., Adv. Func. Mat. 19, 3151-3156 (2009).

[4] F.S. Baumann, et al., J. Electrochem. Soc. 152, A2074-A2079 (2005).

[5] Research supported by ORNL's Shared Research Equipment (ShaRE) User Facility, which is sponsored by the Office of Basic Energy Sciences, U.S. Department of Energy, and by the Materials Science and Engineering Division, Office of Basic Energy Sciences, U.S. Department of Energy.

\begin{tabular}{|c|c|c|c|c|c|}
\hline Region & $\mathrm{keV}$ & $\begin{array}{c}\text { Dose } \\
{\left[\text { ions } / \mathrm{cm}^{2}\right]}\end{array}$ & $\begin{array}{c}\text { Observed } \\
\text { Phase Change }\end{array}$ & $\begin{array}{c}\text { Change in Film } \\
\text { Thickness }\end{array}$ & $\begin{array}{c}\text { Amorphous } \\
\text { Region Width }\end{array}$ \\
\hline A & 30 & $7.5 \mathrm{E} 16$ & Yes & $\sim 10 \mathrm{~nm}$ & $237 \mathrm{~nm}$ \\
\hline B & 30 & $1.5 \mathrm{E} 16$ & No & Negligible & N/A \\
\hline C & 20 & $7.5 \mathrm{E} 16$ & Yes & $\sim 10 \mathrm{~nm}$ & $216 \mathrm{~nm}$ \\
\hline
\end{tabular}

Table 1. The He ion microscope beam energy, dosage, and observations of implantation effects are included in this table.

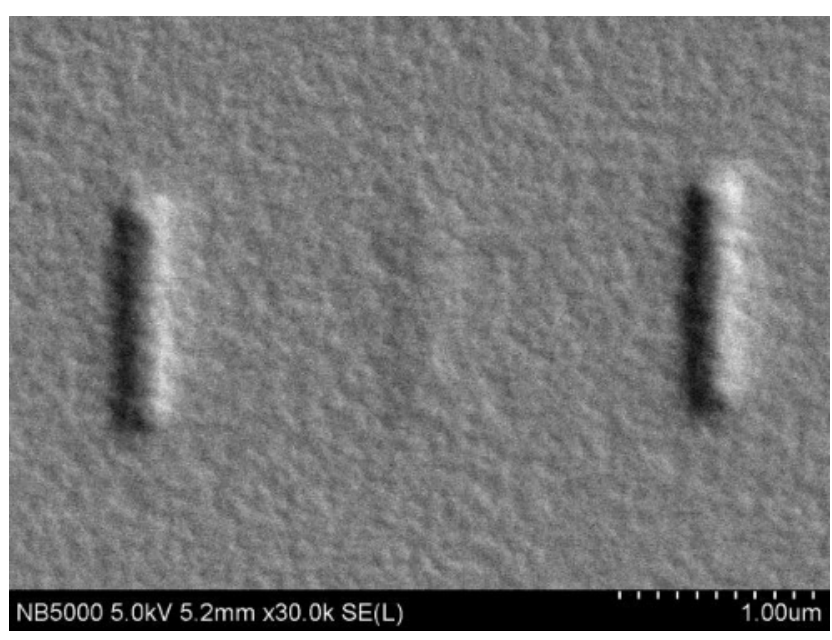

Fig. 1. Implantation of He ions caused swelling of the $\mathrm{LSC}_{113}$ surface in Regions $A$ and $C$. The two regions were the highest dose. Region $B$ was also implanted at $30 \mathrm{keV}$, but the dose was lower, as listed in Table 1.

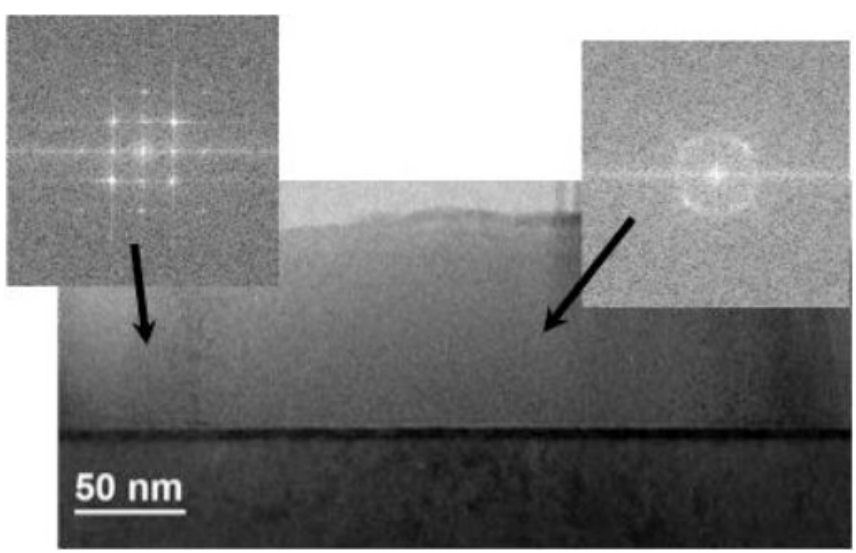

Fig. 3. Cross-sectional STEM bright field image of Region A with associated fft diffractograms (inset) showing the He implantation amorphized the $\mathrm{LSC}_{113}$. Adjacent to the implanted region, the $\mathrm{LSC}_{113}$ film remained crystalline as indicated by the crystalline diffraction pattern.
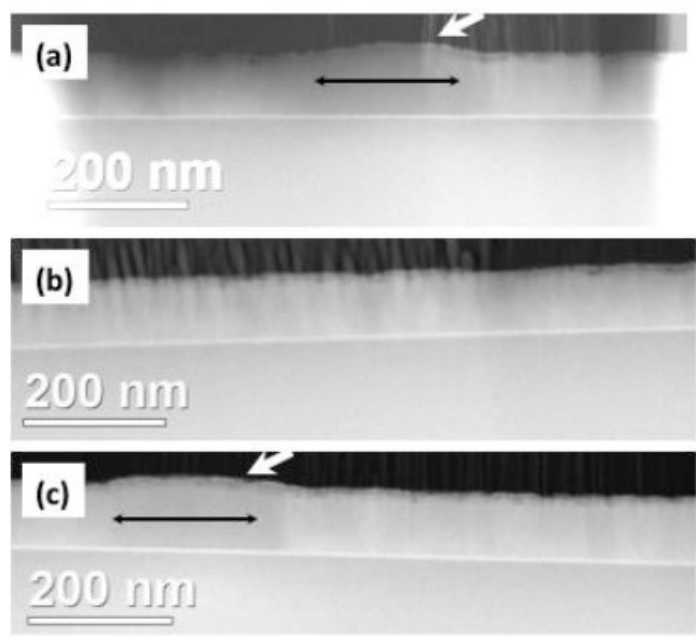

Fig. 2. Cross-sectional HAADF-STEM image of (a) Region A, (b) B, and (c) C, showing the swelling in the implanted regions (white arrow). The widths of the implanted regions are indicated by the black arrows.

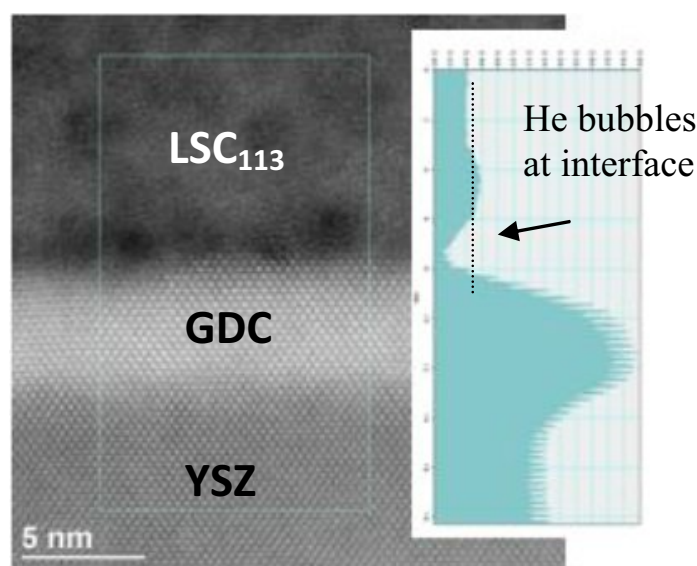

Fig. 4. High magnification HAADF-STEM image of LSC/GDC/YSZ interface in implanted Region A. Arrow denotes the decrease in HAADF intensity caused by pile-up of $\mathrm{He}$ bubbles at the interface. 